\title{
ASSISTÊNCIA À SAÚDE DOS IDOSOS: EQUIPE INTERDISCIPLINAR DE SAÚDE
}

\author{
ELDER HEALTH CARE: INTERDISCIPLINARY \\ HEALTH TEAM
}

\section{ATENCIÓN MÉDICA PARA ANCIANOS: EQUIPO INTERDISCIPLINARIO DE SALUD}

\author{
Maria Clara Duarte Monteiro \\ Maria Manuela Martins ${ }^{2}$ \\ Soraia Dornelles Schoeller ${ }^{3}$ \\ Lucas Antunes ${ }^{4}$
}

Como citar este artigo: Monteiro MCD, Martins MM, Schoeller SD, Antunes L. Assistência à saúde dos idosos: equipe interdisciplinar de saúde. Rev baiana enferm. 2021;35.e36702.

\begin{abstract}
Objetivo: compreender, com base nos discursos da equipa de saúde, o desenvolvimento da assistência aos mais de 65 anos, para se manterem saudáveis, ativos, independentes e autónomos. Método: pesquisa qualitativa com abordagem interpretativa, realizada num Agrupamento de Centros de Saúde do Norte de Portugal. Dados colhidos por entrevista semiestruturada e analisados por meio de análise de conteúdo, por categorias, numa amostra de médicos, enfermeiros e assistentes sociais. Resultados: entre os participantes, 83\% não possuíam formação em gerontologia. Havia focos de avaliação da assistência, em que todos os profissionais estimavam os mesmos dados, mas havia dados necessários ao acompanhamento dos idosos que não eram avaliados por nenhum profissional. A partilha da informação para a assistência, quando ocorria, recaía nas situações de doença ou de alterações do contexto social. Conclusão: achados evidenciaram lacunas no trabalho em equipa desenvolvido pelos profissionais de saúde e sociais, e na assistência no âmbito da Promoção da Saúde.
\end{abstract}

Descritores: Idoso. Promoção da Saúde. Pessoal de Saúde. Equipe de Assistência ao Paciente. Enfermagem.

Objective: to understand, based on the discourses of the health team, the development of care for the elderly aged over 65 years, in order to remain healthy, active, independent and autonomous. Method: qualitative research with interpretative approach, carried out in a Group of Health Centers in Northern Portugal. Data were collected by semistructured interviews and analyzed through content analysis, by categories, in a sample of physicians, nurses and social workers. Results: among the participants, 83\% did not have a gerontology training. There were focuses of care evaluation, in which all professionals estimated the same data, but there were data necessary for the follow-up of the elderly that were not evaluated by any professional. The sharing of information for care, when it occurred, fell into situations of illness or changes in the social context. Conclusion: findings showed gaps in teamwork developed by bealth and social professionals, and in bealth Care Promotion.

Descriptors: Aged. Health Promotion. Health Personnel. Patient Care Team. Nursing.

Objetivo: entender, en base a los discursos del equipo de salud, el desarrollo de la atención a mayores de 65 años, con el fin de mantenerse sanos, activos, independientes y autónomos. Método: investigación cualitativa con enfoque

\footnotetext{
Enfermeira. Mestre em Ciências de Enfermagem. Instituto de Ciências Biomédicas de Abel Salazar da Universidade do Porto. Porto, Portugal. https://orcid.org/0000$0001-8882-5389$

Enfermeira. Doutora em Ciências da Enfermagem. Professora Titular da Escola Superior de Enfermagem. Porto, Portugal. https://orcid.org/0000-0003-I527-9940. Enfermeira. Doutora em Enfermagem. Professora Adjunta da Universidade Federal de Santa Catarina. Florianópolis, Santa Catarina, Brasil. https://orcid.org/00000002-2822-4407.

4 Estudante de Enfermagem. Universidade Federal de Santa Catarina. Florianópolis, Santa Catarina, Brasil. again.lucas@gmail.com. https://orcid.org/0000-0002-5359-4039.
} 
interpretativo, llevada a cabo en un Grupo de Centros de Salud en el Norte de Portugal. Los datos se recogieron por entrevistas semiestructuradas y analizaron a través del análisis de contenidos, por categorías, en una muestra de médicos, enfermeras y trabajadores sociales. Resultados: entre los participantes, el 83\% no tenía formación en gerontología. Hubo focos de evaluación de la atención, en los que todos los profesionales estimaron los mismos datos, pero habia datos necesarios para el seguimiento de las personas mayores que no fueron evaluados por ningún profesional. El intercambio de información para la atención, cuando se produjo, resultó en situaciones de enfermedad o cambios en el contexto social. Conclusión: los hallazgos mostraron lagunas en el trabajo en equipo desarrollado por profesionales de la salud y sociales, y en la atención de promoción de la salud.

Descriptores: Anciano. Promoción de la Salud. Personal de salud. Grupo de Atención al Paciente. Enfermería.

\section{Introdução}

Promover o envelhecimento saudável "[...] requer uma transformação dos sistemas de saúde que substitua os modelos curativos baseados na doença pela prestação de atenção integrada e centrada nas necessidades dos adultos maiores"(1:4). Neste sentido, para o melhor entendimento da problemática em estudo, procurou-se conhecer os idosos da região estudada. Conforme resultados de pesquisa ${ }^{(2)}$ sobre a atividade física habitual de 1.522 idosos pertencentes à área geográfica apoiada por uma Unidade de Cuidados na Comunidade do Concelho, em estudo, verificou-se que $70 \%$ eram inativos ou pouco ativos e $45,7 \%$ possuíam um perfil de estilo de vida negativo. Numa subamostra de 1.322 idosos, "[...] quando se pergunta se usam escadas em vez de elevador 98,5\% dizem não" ${ }^{\text {(2:108-9) }}$. Investigação ${ }^{(3: 7)}$ envolvendo pessoas com mais de 65 anos encontrou que "[...] na maior parte do dia, 99,4\% não realizam atividades físicas moderadas, como caminhar rápido e, no seu lazer, 45,3\% não incluem atividades físicas leves, como passear de bicicleta ou caminhar (duas ou mais vezes por semana)".

Apesar dos numerosos efeitos adversos de um estilo de vida sedentário e dos benefícios, ainda que modestos, do aumento da atividade física, sabe-se, hoje, que a inatividade física continua a ser uma constante entre vários grupos etários da população, sobretudo entre idosos ${ }^{(4)}$, sendo considerado o maior fator de risco comunitário para a saúde em Portugal. Por conseguinte, a tendência crescente do envelhecimento populacional, com agravamento há algumas décadas, "[...] exige uma resposta abrangente da saúde pública”(1:6).
Nas últimas décadas, o índice de envelhecimento da população portuguesa agravou-se, ao registrar, para cada 100 jovens, a existência de 141 idosos em 2014. Segundo dados censitários, este índice era de 102 em 2001 e de 128 em 2011. Pela primeira vez, em Portugal, o número de idosos superou o número de jovens. As projeções da população para 2050 apontam que o número de pessoas com mais de 60 anos ultrapassará o dobro e com mais de 80 anos venha a triplicar $^{(5)}$. Estão associados a esse fator índices de dependência "[...] muitas vezes, acompanhados de situações de fragilidade e de incapacidade, mas frequentemente suscetíveis de prevenção"(6:1) . Relatório da Organização Mundial de Saúde esclarece que comportamentos saudáveis podem prevenir ou retardar problemas de saúde, "[...] principalmente se forem detectados cedo o suficiente [...] entretanto, o mundo está muito longe desses ideais" ${ }^{(1: 6)}$.

As políticas nacionais e internacionais de saúde do idoso enfatizam a preocupação com o aumento da esperança de vida, reconhecendo a necessidade de implementação de intervenções centradas na promoção da autonomia individual e na adoção de medidas de prevenção com foco no envelhecimento ativo e saudável ${ }^{(1,6)}$. No entanto, para se obterem ganhos em saúde, nomeadamente anos de vida com independência, será necessário melhorar as práticas dos profissionais que atuam na área de saúde pública e envelhecimento ${ }^{(6)}$.

Apesar da existência de políticas públicas dirigidas para a saúde do idoso, a literatura evidencia que existem discrepâncias no modelo assistencial em uso pelos profissionais de saúde 
e sociais, verificando-se um desencontro entre as expectativas e a prestação ${ }^{(7-8)}$. A atual prestação de cuidados de saúde a idosos é caracterizada, em vários países, por fragmentação e pouca responsabilização ${ }^{(9)}$. Por isto, a resposta dos sistemas de saúde modernos aos problemas enfrentados por uma população mundial envelhecida tornou-se uma prioridade igualmente importante do ponto de vista social, de saúde e de políticas públicas. É importante que os profissionais entendam a integração de serviços de saúde e sociais dirigida às necessidades multidimensionais dos idosos como um desafio, que requer uma sinergia criada pela boa colaboração multiprofissional $^{(10)}$.

Atendendo a que "[...] num número cada vez maior de países, mais de 1 em cada 5 pessoas tem mais de 60 anos [...] incluir o Envelhecimento Saudável em todas as políticas e em todos os níveis de governo será, portanto, crucial" ${ }^{(1: 22)}$. Por essa razão, acredita-se que este estudo poderá contribuir significativamente para promover a saúde e melhorar a qualidade de vida, de modo que a intervenção recaia no âmbito da promoção da saúde com recurso ao trabalho em equipa interdisciplinar.

Nesse contexto, o ponto de partida do estudo realizado incidiu sobre os resultados de um estudo de investigação realizado num município do Norte de Portugal e respetivo Agrupamento de Centros de Saúde denominado "Modelos em uso na assistência aos Idosos". Para clarificar o caminho da investigação e compreender a problemática em estudo, formulou-se a seguinte questão de pesquisa: Como profissionais de saúde (médicos, enfermeiros e assistentes sociais) desenvolvem o trabalho centrado na assistência à pessoa idosa com vista à promoção da saúde e envelhecimento ativo? A resposta à questão apresentada poderá fornecer subsídios para ampliar o conhecimento acerca da interdisciplinaridade, contribuindo para melhorar a qualidade dos cuidados de saúde aos idosos. Portanto, o presente estudo tem como objetivo compreender, com base nos discursos da equipa de saúde, o desenvolvimento da assistência aos mais de 65 anos, para se manterem saudáveis, ativos, independentes e autónomos.

\section{Método}

Atendendo à natureza específica da problemática em estudo, considera-se essencial o recurso à metodologia qualitativa, com uma abordagem interpretativa. A pesquisa qualitativa tenta interpretar os fenómenos sociais, tais como interações e comportamentos, quanto aos significados que as pessoas the atribuem, pelo que, em função disso, é comumente referida como pesquisa interpretativa ${ }^{(1)}$. Esta abordagem de pesquisa está relacionada com a hermenêutica, "[...] uma vez que enfoca frequentemente, o significado como os indivíduos interpretam o mundo de um dado contexto"(11:205). O descrito corresponde, neste estudo, às experiências vividas pelos profissionais de saúde/socias em contexto de trabalho, onde desenvolvem a sua atividade profissional.

O estudo foi realizado num Agrupamento de Centros de Saúde (ACES) de uma região do Norte de Portugal, durante o ano de 2015. Por tratar-se de um serviço público de saúde, que tem por missão garantir a prestação de cuidados de saúde de proximidade à população de determinada área geográfica, é composto por diversas unidades que integram equipas multiprofissionais. Acresce ainda o facto de os autores conhecerem o contexto dado, os subestudos anteriormente realizados junto da população dos maiores de 65 anos, em que participaram 2.461 idosos, e os resultados que daí emergiram, no que diz respeito à perceção social e de saúde.

A escolha dos sujeitos da pesquisa foi realizada de forma intencional. Para compreender o modelo assistencial em uso para atendimento aos maiores de 65 anos dessa área geográfica, utilizou-se como critérios de inclusão a participação de médicos e enfermeiros com maior incidência de maiores de 65 anos nas suas listas assistenciais e a participação de assistentes sociais que davam assistência a idosos há mais de um ano. Os selecionados acompanhavam diariamente os idosos, ao contrário de outros profissionais que eram consultados pontualmente. Foram excluídos os profissionais que não aceitaram participar do estudo e aqueles com número de idosos inferior a novecentos na sua lista de 
assistência. Utilizou-se o critério de saturação das informações, o qual pressupõe interrupção de inclusão de novos participantes na pesquisa, quando os dados obtidos passam a apresentar redundância ou repetição, não sendo considerado relevante persistir na coleta de dados ${ }^{(11)}$.

A colheita de dados foi realizada por meio de entrevistas, do tipo semiestruturado. Esta modalidade permitiu compreender como os sujeitos (médicos, enfermeiros e assistentes sociais) concebiam o seu mundo e como descreviam essa conceção. Esse tipo de entrevista permite ao entrevistado maior autonomia de resposta, ajudando-o não só a explicitar o seu universo como a torná-lo mais compreensível, possibilitando ao investigador, concomitantemente, a organização e a estruturação do pensamento ${ }^{(11)}$. A amostra da pesquisa procurou um número necessário de participantes para se obter um significado do fenómeno em estudo e resultou dos critérios de inclusão, sendo composta por 8 médicos, 8 enfermeiros e 8 assistentes sociais. A realização de cada entrevista teve a duração aproximada de uma hora.

Para orientação da pesquisa, delinearam-se as seguintes perguntas de investigação: Como descrevem os enfermeiros, os médicos e os assistentes sociais a assistência que prestam? Será que médicos, enfermeiros e assistentes sociais avaliam aspetos diferentes no processo de envelhecimento dos idosos? Como desenvolve a intervenção cada grupo profissional, tendo em vista o envelhecimento saudável, ativo, independente e autónomo?

Os dados colhidos foram submetidos à análise de conteúdo, que teve início desde a primeira entrevista e não apenas após a sua transcrição, momento em que o investigador é inevitavelmente imerso na problemática em estudo, realizando, desde logo, um trabalho reflexivo. A técnica de análise de conteúdo utilizada, seguiu os princípios descritos por Bardin ${ }^{(12)}$, com recurso ao programa informático de análise de dados qualitativos ATLAS.ti, que se revelou muito útil e fiável para a categorização das informações que seriam analisadas. Do processo de análise da informação recolhida nas entrevistas emergiram unidades de significação que originaram duas categorias temáticas: processo de avaliação dos idosos e trabalho em equipa. Esta última, subdivide-se em: meios de partilha da informação de saúde e social e conteúdo ou natureza da informação partilhada.

Para assegurar o anonimato dos sujeitos da pesquisa, as entrevistas foram numeradas de acordo com a sequência em que ocorreram e com a sigla, de acordo com o profissional entrevistado, isto é, EM para médicos, EE para enfermeiros e EAS para assistentes sociais.

De forma a garantir os princípios éticos inerentes ao processo de investigação, o pedido foi remetido à Comissão de Ética para a Saúde da Administração Regional de Saúde do Norte, sendo aprovado com o № 19/2015. Todos os sujeitos que concordaram em participar do estudo assinaram o Termo de Consentimento Livre e Esclarecido e confirmaram o conteúdo final das entrevistas.

\section{Resultados}

A pesquisa desenvolveu-se com 24 participantes, sendo um homem e 23 três mulheres, destacando-se a totalidade dos enfermeiros e dos assistentes sociais do sexo feminino. As idades dos três grupos profissionais variou de 30 anos ao máximo de 60 anos, com predominância da faixa etária de 31 a 40 anos nos grupos dos assistentes sociais e dos enfermeiros. No grupo dos médicos, prevaleceu a faixa etária dos 51 a 60 anos. No que concerne à formação específica em gerontologia, 20 dos profissionais em estudo, não a possuíam. No âmbito do processo de avaliação, as áreas de intervenção de cada um dos profissionais junto aos idosos na primeira consulta e as áreas de intervenção comuns entre todos estão apresentadas na Figura 1. 
Figura 1 - Avaliação assistencial do idoso

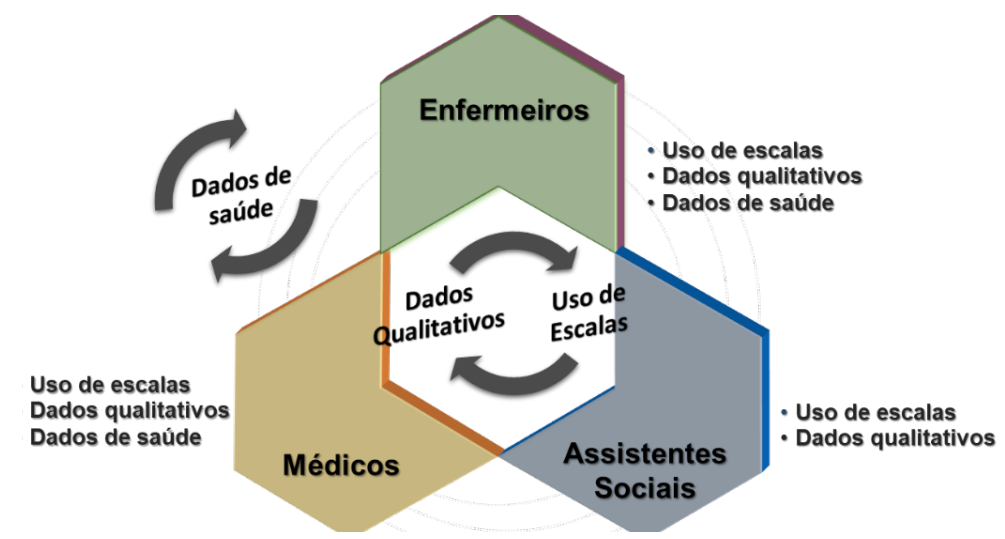

Fonte: Elaboração própria.

Dos discursos dos profissionais de saúde e sociais emergiram áreas comuns da avaliação inicial do idoso, como o uso de escalas, sobretudo o índice de Barthel.

[...] aplicamos também uma escala da dependência, que é a escala de Barthel, que neste momento é mediante essa escala que nós fazemos uma vigilância mais ou menos apertada ao utente. (EE2).

Escala Barthel, para saber se independente ou não.(EM2).

[...] temos numa fase inicial na altura da candidatura, a escala de Barthel, que adotamos até para o nivel de dependência que normalmente é fisica que essa escala dâ [...] (EAS4).

Emergiu ainda dos discursos dos participantes a recolha de dados qualitativos, tal como o contexto sociofamiliar.

Sim, temos sempre que avaliar o grau de dependência do utente, isto na primeira visita, a consulta de enfermagem, as condições, com quem vive, quem é o prestador de cuidados, se está num apoio domiciliário [...] (EE3)

Depois também há todo aquele contexto social, se a pessoa vive sozinha, se está acompanhada, se tem apoio de vizinhos, se tem de amigos ou parentes. (EM7).

Nós fazemos a avaliação da situação familiar e económica. (EAS8).

Entre enfermeiros e médicos, era comum a colheita de dados de saúde, como seria expectável, porém focada na doença/dependência, patologias, risco de úlcera de pressão, entre outros.

Se o doente está autónomo, se o doente não apresenta nenbum défice nem nenhuma queixa a nivel motor nem cognitivo [...] se eventualmente tem algum tipo de alteração é adaptado. (EM3).

Vejo quais são os problemas do utente, levanto os focos e completo uma avaliação inicial normal, independentemente se tem mais de 65 anos. (EE2).
Entretanto, apenas os enfermeiros referiram utilizar o SClínico como recurso tecnológico de suporte para realizar a avaliação.

Nós cá, no serviço, trabalhamos com o SClínico em termos informáticos. Em termos de saúde do idoso, o próprio programa já nos dá. Nós abrindo o programa de saúde do idoso a partir dos 65 é obrigatório num doente que nos chegue com mais de 65 anos, ele entra-nos logo nos autocuidados, autocuidado higiene, vestuário, transferência. Nós temos que colocar se tem algum tipo de dependência ao realizar esse tipo de autocuidado, mas trabalhamos mais nessas áreas [...] (EE6).

Percebeu-se, nos discursos dos enfermeiros, que a avaliação inicial realizada dirigia-se para responder ao programa de saúde incorporado no sistema de informação clínico, podendo, deste modo, tornar-se redutor para uma avaliação multidimensional, com foco na dependência para o autocuidado. Os médicos e assistentes sociais priorizavam, na sua avaliação, aspetos cognitivos, com recurso ao Mini Mental State Examination (MMSE), conforme exemplificado.

Sim, passamos normalmente o mini mental. (EAS5).

Cognitivos. É o mini mental que a gente mais usa. (EM3).

Já os enfermeiros priorizavam aspetos físicos, utilizando a escala de Norton ou Braden:

[...] e depois fazemos sempre uma avaliação de uma escala de Norton, ou de uma escala de Braden para depois atribuir um plano de cuidados àquele doente e família. (EE3).

No que concerne ao trabalho em equipa, emergiram duas áreas relevantes da comunicação estabelecida entre os profissionais de saúde e sociais, subsequentes à assistência aos idosos, conforme apresentado na Figura 2: meios de partilha da informação e conteúdo da informação partilhada. 
Figura 2 - Comunicação interdisciplinar: informação partilhada e meios de partilha da informação

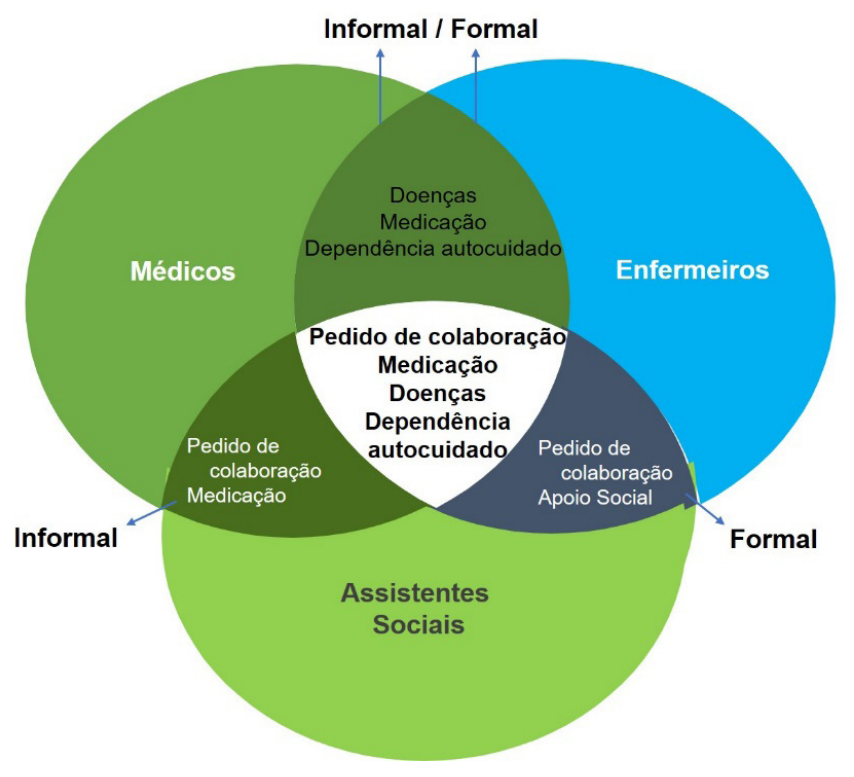

Fonte: Elaboração própria.

Entre os profissionais de saúde e sociais do estudo, todos recorriam aos dois meios de comunicação, embora o modo informal se sobrepusesse ao modo formal para médicos, sobretudo o diálogo.

[...] se forem realmente alterações que eu faça e que eu ache que tenha alguma importância para a parte de enfermagem ou alguma alteração do contexto social, pois, com certeza, que comunico. (EM7).

Para assistentes sociais, a conversa pessoal ou telefónica predominou:

[...] ligamos diretamente ao centro de saúde, falamos com o enfermeiro [...] (EAS5).

Quanto aos enfermeiros, recorriam mais ao meio formal, fazendo uso do sistema de informação clínico $\left(\right.$ SClínico ${ }^{\circledR}$ ) ou ainda por carta escrita e/ou protocolo de ligação.

Nós agora também temos um novo sistema que é o SClinico, que os nossos dados são observáveis pela médica e nós também conseguimos observar a parte médica [...] (EE5),

Além dessa partilha de informação verbal (não documentada), ocorriam ainda os momentos em que os profissionais de saúde e sociais, no seu quotidiano, apenas a realizavam perante algum motivo que a justificasse. Os motivos pelos quais os assistentes sociais requeriam colaboração de um profissional de saúde estavam relacionados com situações de doença, para obter o complemento de dependência ou mesmo para esclarecer dúvidas de saúde/doença. De igual modo acontecia com os enfermeiros.

Por exemplo, a situação em que há doença. (EAS5).

[...] no sentido de obter um relatório médico, geralmente precisamos de um relatório médico. Aqui ninguém vai para um lar sem saber que tipo de patologias apresenta. (EAS7).

[...] é só se houver alguma coisa bizarra ou anormal [...] se for uma situação normal que o utente está bem, não temos esse hábito. (EE5).

O médico, sendo o profissional que menos partilhava a informação, também solicitava a colaboração de outros profissionais, mas apenas de forma esporádica, em situação de doença ou de alteração do estado de saúde.

Partilhamos a evolução, o seu controlo de doenças, se está a fazer bem ou não está, se surgiu algum problema de novo. A enfermeira também partilha comigo, se alguma coisa não está bem, porque, às vezes, eles vêm à consulta de enfermagem e não vêm à consulta médica. Por exemplo, quando alguma coisa está descontrolada em termos de diabetes, de HTA [Hipertensão Arterial], se o utente parece assim um bocadinho desorientado. (EM1).

Os discursos partilhados entre médicos e enfermeiros eram, sobretudo, relativos às doenças em geral, mas também à medicação e à dependência no autocuidado. Entretanto, os enfermeiros partilhavam com o médico, para a dependência no autocuidado. 
[...] utente dependente, falamos dos problemas de saúde, dos crónicos. É uma doente hipertensa, diabética ou então de problemas agudos, teve internada por uma infeção respiratória, tem estas lesões [...] (EE2).

Já os médicos partilhavam as doenças, sobretudo com os enfermeiros.

Cá no centro de saúde, aqueles que têm doenças crónicas, acabamos também por partilhar muita informação [...] (EM6).

No que se refere aos discursos que os médicos partilhavam com os assistentes sociais, incidiam nos pedidos de colaboração para intervenção, nomeadamente em situações de abandono do idoso e/ou de outra necessidade de apoio.

Com a assistente social já aconteceu utentes meus que se descobre que estão abandonados no domicílio. Então, ativamos os serviços sociais. (EM7).

Com a assistente social nem por isso, é outra situação em que há necessidade de apoio ou de intervenção, porque senão é muito raro. Até hoje acho que, mesmo de idosos, não tive que recorrer. (EM6).

Constatou-se que os assistentes sociais partilhavam com os médicos a medicação habitual do idoso, seguido também do pedido de colaboração, no caso de maus-tratos ou para solicitar um relatório médico, com o intuito de integração em lar.

Geralmente precisamos de um relatório médico. Aqui ninguém vai para um lar sem saber que tipo de patologias apresenta [...] a terapêutica que está a fazer. (EAS7).

Relativamente aos discursos dos enfermeiros com os assistentes sociais, incidiam no pedido de colaboração para aquisição de medicamentos e de apoio social por más condições habitacionais,

Um doente que nos chegue cá e nós nos deparamos que necessite de algum apoio social, se tem alguma dificuldade na aquisição de alguma medicação, nós também encaminhamos para apoio social [...] (EE6).

Os assistentes sociais revelaram, dos seus discursos com os enfermeiros, sobretudo a capacidade funcional do idoso, seguindo-se os pedidos de colaboração para aquisição de ajudas técnicas, de complemento de dependência ou apoio em situações de maus-tratos,

[...] acho que já não tem capacidade para algumas tarefas de vida diária e aí a enfermeira diz-me se sim ou se não. (EAS3).

[...] nas questões de maus-tratos, eu articulo sempre. A avaliação é feita por mim, pela colega psicóloga e pelos serviços de saúde. Sempre estes três. (EAS8).
[...] imagina se eu acho que a pessoa precisa do complemento de dependência, portanto peço opinião se valeria a pena. Por exemplo, o idoso ser encaminhado para requerer o complemento de dependência. (EAS3).

\section{Discussão}

Em Portugal, "Ao longo dos anos, a taxa de feminização [...] tem apresentado uma tendência geral de crescimento. Em 2018 [...] fixou-se nos 76,5\%, o que está acima da taxa da administração pública no global $(60,2 \%)^{\text {"(13:57) }}$. À semelhança desse estudo, constatou-se que a amostra é constituída maioritariamente por mulheres (1 homem e 23 mulheres).

Estudo aponta que o pessoal de enfermagem é o que mais contribui para essa taxa, superando os demais grupos profissionais ${ }^{(13)}$. No caso dos assistentes sociais deste estudo, tal não aconteceu, pois todos eram do sexo feminino. A média etária encontrada foi de 42,4 anos, sendo a idade mínima de 30 anos e a máxima de 60 anos, correspondendo respetivamente a enfermeiros e médicos. A maioria dos profissionais $(83,3 \%)$ não tem formação em gerontologia, o que pode interferir na assistência interdisciplinar aos idosos. Nesta perspetiva, existe evidência que indica "[...] diferença entre os processos de trabalho das profissões da área médica (com foco nas especializações) e o Serviço Social com formação generalista" ${ }^{(14: 97)}$, comprovando que a natureza do trabalho com idosos exige do profissional a procura de formação contínua e necessidade de constante atualização.

\section{Processo de Avaliação dos Idosos}

Entende-se por avaliação, a recolha de diversos dados relativos ao idoso ao nível físico, mental, funcional e social efetuada por equipa multiprofissional quando da primeira consulta, com o objetivo de estabelecer e coordenar planos de cuidados, serviços e intervenções que respondam aos seus problemas, às suas necessidades e às suas incapacidades. A avaliação das distintas dimensões possibilita uma intervenção mais completa e adequada dos profissionais e, consequentemente, melhor qualidade de vida para o idoso. 
Para os enfermeiros, a avaliação corresponde à primeira etapa do Processo de Enfermagem, que se revela de extrema importância para a identificação de necessidades em cuidados de enfermagem. O índice de Barthel é o instrumento o mais comumente usado para avaliar se o idoso é capaz de desempenhar determinadas atividades de modo independente, compreendendo dez atividades básicas da vida diária (ABVD): comer, higiene pessoal, uso de sanitário, tomar banho, vestir e despir, controlo de esfíncteres, deambular, transferência da cadeira para a cama, subir e descer escadas. Ainda centrados nos aspetos físicos, os enfermeiros aplicam a escala de Norton ou de Braden na avaliação da saúde dos idosos. Os referidos instrumentos são frequentemente utilizados na prática profissional desses profissionais, pois a lesão por pressão é um foco de atenção altamente sensível aos cuidados de enfermagem. No entanto, admite-se que esta prática não se encontra sistematizada em todas as instituições de saúde ${ }^{(15)}$.

Estudos nacionais e internacionais ${ }^{(16-17)}$ reforçam o conhecimento sobre a eficácia de uma avaliação holística e multidimensional do idoso, contemplando a avaliação clínica, física, mental, funcional, social, nutricional, entre outras, com o recurso de vários instrumentos. "Todas as evidências sugerem que beneficia tanto os indivíduos saudáveis quanto aqueles com deficiências significativas e múltiplas comorbilidades",(17:7). Entende-se que essa avaliação revela também uma mudança de paradigma, dado que, atualmente, não é considerada para pessoas saudáveis e está ainda muito dirigida para identificar problemas médicos, mentais e funcionais de idosos com fragilidade ${ }^{(16)}$. Outros achados destacam incidir na avaliação do idoso com alterações cognitivas significativas, para além da morbilidade e da funcionalidade ${ }^{(16)}$. Pressupondo esta avaliação multidimensional realizada por uma equipa constituída por médico, enfermeiro e assistente social, entre outros, os Cuidados de Saúde Primários constituem locais privilegiados para uma assistência interdisciplinar aos idosos e promotora de saúde.

A avaliação global do idoso é uma estratégia que facilita uma abordagem multifatorial apoiada em um processo estruturado, com recurso a escalas ou questionários de avaliação desenvolvidos nas últimas décadas e validados cientificamente.

\section{Trabalho em Equipa}

A complexidade dos cuidados em saúde atuais exige que "[...] o trabalho em equipa (TE) seja sistematicamente referido como um factor humano fundamental para a qualidade do cui-

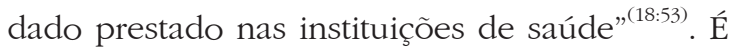
seu propósito eliminar o pensamento fragmentado e promover o cuidado integrado. Este é descrito como um conjunto bem organizado de serviços e processos de assistência voltado para as necessidades multidimensionais dos idosos, que concede ao desempenho articulado da equipa multiprofissional melhores resultados do que os desempenhos individuais ${ }^{(10)}$. Para fortalecer os comentários expostos anteriormente, defende-se que a mudança para a assistência integral exige a reestruturação ao nível dos serviços, bem como a reformulação do trabalho desenvolvido pelas equipes de saúde com enfoque na colaboração interdisciplinar. Importa considerar também que o trabalho em equipa e a comunicação estão intimamente ligados à segurança dos doentes ${ }^{(7)}$.

\section{Meios de partilha da informação de saúde} e social

A informação é muito valiosa para a prestação dos cuidados de saúde com qualidade e segurança, constituindo uma condição basilar dos processos de gestão dos cuidados de enfermagem. Assim, o acesso rápido e facilitado aos dados tem contribuído para melhorar o processo de tomada de decisão clínica ${ }^{(19)}$, assumindo extrema relevância para a comunicação eficaz entre médicos, enfermeiros e assistentes sociais que trabalham em equipa. Os meios de comunicação utilizados pelos participantes divergem entre informais e formais, com implicações distintas na prática de cuidados, traduzindo-se em mais ou menos ganhos para a saúde dos utentes. Resultados semelhantes foram encontrados noutro estudo desenvolvido em Ontário ${ }^{(20)}$. 
Os meios informais de comunicação, a exemplo das conversas pontuais, refletem a tradicional conversa de corredor sobre assuntos de interesse para o profissional naquele momento. Os meios formais de comunicação são constituídos pela documentação sistemática da atividade de cada profissional no processo clínico do utente (processo único), acessível a todos os profissionais em situações relevantes da prestação de cuidados (Registo de Saúde Eletrónico) ou, ainda, pelas reuniões periódicas da equipa, além de atas de reuniões, memorandos, comunicação assistida por computador e registos de comunicação $^{(20)}$.

As reuniões interprofissionais regulares são consideradas como um meio essencial para a construção de uma boa comunicação em equipa $^{(20-21)}$. Outro sistema de informação é o SClínico, interoperável, capaz de garantir qualidade e continuidade de cuidados ${ }^{(22)}$. A necessidade de melhorar o desempenho da equipa multidisciplinar, para homogeneizar as práticas e a informação recolhida nacionalmente, é reiterada pelos Serviços Partilhados do Ministério da Saúde de Portugal com o desenvolvimento do SClínico ${ }^{\circledR}$. Esse sistema tem tornado a atuação dos profissionais de saúde mais eficaz e eficiente, possibilitando melhor apoio, assistência e acompanhamento ao utente.

Apesar disso, continua-se a verificar uma fragmentação da documentação dos cuidados de saúde prestados pela equipa multiprofissional, que em nada preconiza a integralidade do indivíduo. Estudo nacional relatou, em relação às falhas na comunicação, que "[...] 50\% dos casos tinham inexistência de comunicação eficaz entre os profissionais de saúde, de forma a garantir a continuidade informacional nos cuidados de saúde" ${ }^{(23: 5)}$. Desse modo, admite-se a necessidade de uma comunicação efetiva entre os setores de cuidados, para uma atuação conjunta, capaz de permitir troca de saberes. Entretanto, apesar da evolução das ciências médicas com enfoque nos aspetos de prevenção e promoção da saúde, em desfavor dos aspetos puramente curativos, encontra-se ainda o predomínio do modelo biomédico na partilha da informação em uso entre os profissionais de saúde e sociais.
Corrobora esse entendimento, a seguinte afirmação: "[...] os seguidores deste modelo têm uma atitude redutora, de quem vê a doença, o órgão e desvaloriza a tradução subjetiva da doença, com as relações pessoais, familiares, sociais e culturais” ${ }^{, 24: 36)}$. Isso se poderá verificar nos discursos sobre o conteúdo da informação partilhada, que será determinante para a sua disseminação (ou não) entre todos. Este modelo está em discordância com práticas promotoras de um envelhecimento ativo, autónomo e saudável ${ }^{(1,6-7)}$. Outros autores ${ }^{(22: 91)}$ partilham a mesma opinião, admitindo que "[...] são imperativos estudos que enfoquem este fenómeno, da partilha da informação, entre os dois grupos profissionais mais decisivos em saúde (médicos e enfermeiros)" estabelecendo-se uma nova relação entre eles, distante do modelo biomédico, baseada na interdisciplinaridade e não na multidisciplinaridade. Esta mudança permite maior diversidade das ações e busca permanente de consenso, o que requer uma comunicação horizontal entre os elementos de uma equipa. Numa perspetiva interdisciplinar, a abordagem do problema seria vista conjuntamente, bem como a busca de soluções criativas para resolvê-lo.

\section{Conteúdo ou natureza da informação partilhada}

A informação diz respeito a dados que foram interpretados, organizados e estruturados, resultantes da documentação da assistência nos sistemas de informação em saúde, contribuindo para a promoção da continuidade dos cuidados, mediante a sua utilização pelos diversos profissionais de saúde. Estudo ${ }^{(22: 88)}$ verificou que o "[...] conteúdo da informação recolhida, processada e documentada pelos enfermeiros, relevante para efeitos do exercício profissional dos médicos, organiza-se em três categorias: parâmetros de vigilância, medicação e atitudes terapêuticas, e dados intercorrentes". Neste estudo, evidenciaram-se, quanto às áreas de informação comuns entre todos os profissionais de saúde e social: os pedidos de colaboração, a medicação, as patologias em geral e a dependência no autocuidado. Estas intervenções em uso na assistência 
aos idosos fazem emergir reflexões e questionamentos, tais como: Os idosos não recebem orientações promotoras de saúde? Os profissionais de saúde e sociais dirigem a sua atenção tendo em vista o envelhecimento saudável, ativo, autónomo e independente? Médicos, Enfermeiros e Assistentes Sociais, dirigem todos a sua intervenção para as mesmas áreas?

Por outro lado, a carência de estudos sobre esta temática estimula a criação e a reformulação de políticas públicas que garantam uma comunicação interdisciplinar padronizada. Desse modo, não é possível falar sobre qualidade em saúde sem fazer referência à qualidade da interação e da comunicação entre os profissionais responsáveis pelo cuidado e pela segurança do doente.

A falha de comunicação está entre as principais causas de eventos adversos à saúde em âmbito internacional. Até 70\% desses eventos ocorrem entre os profissionais de saúde ${ }^{(23)}$. Pesquisa aponta diferenças hierárquicas, poder e conflitos entre os principais motivos encontrados, que podem inibir os elementos da equipa interdisciplinar. Salienta-se ainda que a dificuldade de articulação em equipa está geralmente relacionada com as distintas formações de base, ideias e papéis de cada grupo profissional ${ }^{(21)}$. Frente a estas questões, o desenvolvimento de programas de formação-ação sobre estratégias para a melhoria da comunicação em equipe, compreendendo todos os elementos abrangidos, torna-se cada vez mais premente.

A comunicação é também importante para a ação dos assistentes sociais, pois são esses profissionais os que mais solicitam a colaboração dos demais, o que revela a necessidade de interligação constante do serviço social com os profissionais de saúde. Estudo realizado na Finlândia ${ }^{(10)}$ admite a necessidade de reforçar a articulação entre os profissionais sociais e de saúde, para que, em equipa, atendam melhor às necessidades dos idosos. Todavia, estudo ${ }^{(18)}$ demonstra que o envolvimento das instituições de saúde na formalização e no apoio de atividades interdisciplinares muito contribui para a existência de equipas de trabalho funcionais. Do mesmo modo, pesquisa admite que a liderança é eficaz na promoção do entendimento mútuo entre as várias profissões ${ }^{(21)}$.

Pelo exposto, cabe a reflexão do necessário empenho das instituições de saúde, dos gestores e dos profissionais da equipa para a resolução de um problema comum. O desenvolvimento desta cultura interdisciplinar refletirá na qualidade dos processos assistenciais e, consequentemente, na segurança dos clientes.

Relata-se como limitação do estudo o fato de todos os profissionais serem de uma só região, o que circunstancia uma realidade limitada.

\section{Conclusão}

Os profissionais do estudo não demonstraram intervir de forma coordenada e centrada nas especificidades dos idosos. Referiram ainda não ter, na sua maioria, formação em gerontologia/ geriatria.

Sobre o processo de avaliação dos idosos, há dados que todos os profissionais priorizam e outros que ninguém avalia. Todos recolhem dados sobre o estado funcional, isto é, a dependência para a realização de atividades de vida diária e sobre o estado social e o contexto sociofamiliar, mas não contemplam os idosos saudáveis. O foco das ações dirige-se apenas aos idosos doentes/dependentes de terceiros.

No que se refere à comunicação interdisciplinar, aspeto particularmente relevante na assistência aos idosos, os resultados obtidos denotam que os meios de partilha da informação entre médicos, enfermeiros e assistentes sociais que trabalham em equipa são distintos e surgem apenas de modo esporádico, isto é, quando existe um motivo. Este resultado revela a fragmentação do processo assistencial interdisciplinar, que compromete a qualidade e a continuidade de cuidados.

Merece destaque o conteúdo da informação partilhada em equipa, resultante das intervenções individuais que incidem na medicação habitual, doenças, dependência no autocuidado e pedidos de colaboração, com enfoque das respetivas intervenções para o tratamento da doença ao invés da promoção da saúde. Entretanto, o 
facto de os assistentes sociais solicitarem mais a colaboração de outros profissionais evidencia a necessidade de articulação constante entre o serviço social e o serviço de saúde.

Pelo exposto, considera-se que urge envolver as diferentes profissões, criar sinergias entre as diferentes áreas de conhecimento, desenvolver as skillmix para uma mudança de paradigma na assistência aos idosos no âmbito da promoção da saúde.

A carência de estudos sobre esta temática estimula a criação e reformulação de políticas públicas que garantam um sistema uniformizado e interdisciplinar de avaliação da pessoa idosa.

Compreender o modelo assistencial em uso, na perspetiva dos profissionais de saúde e sociais, contribui para melhorar o trabalho em equipa interdisciplinar e acentuar as suas potencialidades, proporcionando o desenvolvimento de estratégias que aperfeiçoem, cada vez mais, a assistência aos idosos.

\section{Fonte de Financiamento:}

Esta pesquisa foi parcialmente apoiada pelo financiamento do município de Vila Nova de Famalicão.

\section{Colaborações:}

1 - concepção, projeto, análise e interpretação dos dados: Maria Clara Duarte Monteiro, Maria Manuela Martins, Soraia Dornelles Schoeller;

2 - redação do artigo e revisão crítica relevante do conteúdo intelectual: Maria Clara Duarte Monteiro, Maria Manuela Martins, Soraia Dornelles Schoeller e Lucas Antunes;

3 - aprovação final da versão a ser publicada: Maria Clara Duarte Monteiro, Maria Manuela Martins e Soraia Dornelles Schoeller.

\section{Referências}

1. Organização Mundial da Saúde. Relatório Mundial de Envelhecimento e Saúde [Internet]. Genebra; 2015 [cited 2019 Mar 19]. Available from: http:// apps.who.int/iris/bitstream/10665/186468/6/ WHO_FWC_ALC_15.01_por.pdf?ua=1
2. Cardoso T, Martins MM, Monteiro MC. Unidade de Cuidados na Comunidade e promoção da saúde do idoso: um programa de intervenção. Rev Enf Ref. 2017;serIV(13):103-14. DOI: http:// dx.doi.org/10.12707/RIV16071

3. Martins M, Monteiro C, Martinho J, Guerra-Martìn MD, Alves I, Vieira M. Atividade física nos mais de 65 anos e a Promoção da Saúde. Actas Gerontol [Internet]. 2016 [cited 2018 Sep 24];2:1-10. Available from: https:// www.researchgate.net/publication/295078234_ Atividade_fisica_nos_mais_de_65_anos_e_a_ Promocao_da_Saude

4. Escalante Y. Actividad física, ejercicio físico y condición física en el ámbito de la Salud Pública. Rev Esp Salud Pública [Internet]. 2011 [cited 2017 Nov 15];85(4):325-8. Available from: http://scielo.isciii.es/scielo.php?script=sci_ arttext\&pid=S1135-57272011000400001

5. Portugal. Instituto Nacional de Estatística. Dia Mundial da População - 11 de julho. Envelhecimento da população residente em Portugal e na União Europeia [Internet]. Lisboa (PT); 2015 [cited 2020 Apr 27]. Available from: https:// www.ine.pt/xportal/xmain?xpid=INE\&xpgid=ine_ destaques\&DESTAQUESdest_boui $=224679354 \&$ DESTAQUES modo $=2 \&$ xlang $=$ pt $\&$ xlang $=$ pt\&xlang=pt

6. Portugal. Serviço Nacional de Saúde. Direção-Geral da Saúde. Documentos e Publicações. Programa Nacional para a Saúde das Pessoas Idosas [Internet]. Lisboa (PT); 2006 [cited 2020 May 3]. Available from: https://www.dgs.pt/ documentos-e-publicacoes/programa-nacionalpara-a-saude-das-pessoas-idosas-pdf.aspx

7. Portugal. Ministério da Saúde. DireçãoGeral da Saúde. Plano Nacional de Saúde: Revisão e Extensão a 2020 [Internet]. Lisboa (PT); 2015 maio [cited 2015 Jun 2]. Available from: https://drive.google.com/drive/folders/ 1eozNFyshkj7xOnuBZfL0aKMtVTvwNKGT?usp =sharing

8. Young HM, Siegel EO, McCormick WC, Fulmer T, Harootyan LK, Dorr DA. Interdisciplinary collaboration in geriatrics: advancing health for older adults. Nurs Outlook. 2011;59(4):243-50. DOI:https://doi.org/10.1016/j.outlook.2011.05.006

9. Eloranta S, Arve S, Routasalo P. Multiprofessional collaboration promoting home care clients' personal resources: perspectives of older clients. Int J Older People Nurs. 2008 Jun;3(2):88-95. DOI: https://doi.org/10.1111/j.1748-3743.2007.00105.x 
10. Boeckxstaens P, De Graaf P. Primary care and care for older persons: position paper of the European Forum for Primary Care. Qual Prim Care [Internet]. 2011 [cited 2020 Nov 1];19(6):369-89. Available form: https://pubmed.ncbi.nlm.nih. gov/22340900/

11. Streubert HJ, Carpenter DR. Investigação qualitativa em enfermagem: avançando o imperativo humanista. 5a ed. Loures: Lusociência; 2002.

12. Bardin L. Análise de conteúdo. 5a ed. Lisboa: Edições 70; 2009.

13. Portugal. Serviço Nacional de Saúde. Relatório Social do Ministério da Saúde e do Serviço Nacional de Saúde [Internet]. Lisboa (PT); 2018 [cited 2019 Sep 28]. Available from: https://www.sns.gov.pt/ wp-content/uploads/2019/09/Relat\%C3\%B3rioSocial-MS_SNS-2018-002.pdf /

14. Silveira R, Silva E. O trabalho do/a Assistente Social na Unidade de Terapia Intensiva (UTI): a (in) visibilidade de suas ações $\mathrm{x}$ os processos de trabalho em equipe. Textos Contextos. 2018;17(1):97-114. DOI: $10.15448 / 1677-9509.2018 .1 .27325$

15. Portugal. Serviço Nacional de Saúde. DireçãoGeral da Saúde. Plano Nacional para a Segurança dos Doentes 2015-2020 [Interrnet]. Lisboa (PT); 2015 [cited 2015 Sep 22]. Avaiable from: https:// www.dgs.pt/qualidade-e-seguranca/segurancados-doentes/plano-nacional-para-a-segurancados-doentes-2015-2020.aspx

16. Ellis G, Gardner M, Tsiachristas A, Langhorne P, Burke O, Harwood RH, et al. Comprehensive geriatric assessment for older adults admitted to hospital. Cochrane Database Syst Rev. 2017;9(9):CD006211. DOI: 10.1002/14651858. CD006211.pub3

17. López MVG, Bastante MM, Torres LM, Cantero ADP. Cuidados básicos para la edad avanzada. Spain: Grupoparadigma; Editorial DAE; 2015. Serie Cuidados Básicos, Geriatria, Gerontologia y enfermería geriátrica.
18. Santos MC, Grilo AM, Andrade G, Guimarães T, Gomes A. Comunicação em saúde e a segurança do doente: problemas e desafios. Rev Portuguesa Saude Pub [Internet]. 2010 [cited 2020 May 1];(10):47-57. Avaliable from: http://hdl. handle.net/10400.21/3120

19. Pereira F. Informação e qualidade do exercício profissional dos enfermeiros. Coimbra: Formasau; 2009.

20. Brown JB, Lewis L, Ellis K, Stewart M, Freeman TR, Kasperski MJ. Mechanisms for communicating within primary health care teams. Can Fam Physician [Internet]. 2009 [cited 2018 Aug 26];55(12):1216-22. Available from: https:// pubmed.ncbi.nlm.nih.gov/20008604/

21. Asakawa T, Kawabata H, Kisa K, Terashita T, Murakami M, Otaki J. Establishing community-based integrated care for elderly patients through interprofessional teamwork: a qualitative analysis. J Multidiscip Healthc. 2017;10:399-407. DOI: 10.2147/JMDH.S144526

22. Mota L, Pereira F, Sousa P. Sistemas de Informação de Enfermagem: exploração da informação partilhada com os médicos. Rev Enf Ref. 2014;serIV(1):85-91. DOI: 10.12707/RIII12152

23. República Portuguesa. Serviço Nacional de Saúde. Direção-Geral da Saúde. Norma № 001/2017, de 8/2/2017. Comunicação eficaz na transição de cuidados de saúde [Internet]. Lisboa (PT); 2017 [cited 2017 Jul 19]. Available from: http://nocs.pt/wp-content/uploads/2017/10/ i023296.pdf

24. Sequeira C. Introdução à prática clínica: do diagnóstico à intervenção em enfermagem de saúde mental e psiquiátrica. Coimbra: Quarteto; 2006.

Recebido: 5 de maio de 2020

Aprovado: 14 de setembro de 2020

Publicado: 13 de abril de 2021

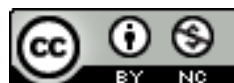

A Revista Baiana de Enfermagem utiliza a Licença Creative Commons - Atribuição-NãoComercial 4.0 Internacional. https://creativecommons.org/licenses/by-nc/4.0/ Este artigo é de acesso aberto distribuído sob os termos da Licença Creative Commons (CC BY-NC). Esta licença permite que outros remixem, adaptem e criem a partir do seu trabalho para fins não comerciais. Embora os novos trabalhos tenham de lhe atribuir o devido crédito e não possam ser usados para fins comerciais, os usuários não têm de licenciar esses trabalhos derivados sob os mesmos termos. 